\title{
An efficient computerized testing method for the capuchin monkey (Cebus apella): Adaptation of the LRC-CTS to a socially housed nonhuman primate species
}

\author{
Theodore A. Evans, Michael J. Beran, Betty Chan, Emily D. Klein, \\ and Charles R. Menzel \\ Georgia State University, Decatur, Georgia
}

\begin{abstract}
Even with advances in automated testing techniques, the capuchin monkey (Cebus apella) can be a difficult species to test in the laboratory, given its social/behavioral tendencies and typical activity pattern. Laboratories that maintain social colonies of capuchin monkeys are able to separate and test individuals, but the process can be very effortful and time consuming, and the resulting data can be modest in quantity. The present article describes procedures and apparatuses that were used to train a colony of computer-naive capuchin monkeys to quickly and reliably isolate themselves from group members and interact with a computerized test system in order to produce a large volume of data. Several elements that were important in motivating the monkeys to participate are discussed.
\end{abstract}

Despite developments in computer testing technology, psychological testing of individual nonhuman primates (NHPs) can be a difficult process, especially when animals are socially housed. Captive NHPs are often kept in social groups to mimic the natural sociality that comprises a large part of primate psychology. Additionally, NHPs are studied in groups because their social behavior, in itself, can produce psychological phenomena as interesting as some of the cognitive, perceptual, and psychomotor processes that are often of primary concern to NHP investigators. However, if one is interested in testing individual members of the social group, social housing can become an obstacle, compromising an experimenter's ability to isolate and motivate animals to participate in tasks.

Computerized testing of capuchin monkeys (Cebus apella), in particular, can be difficult because of their species-typical behavioral repertoire. Capuchins are naturally arboreal (Fortman, Hewett, \& Bennett, 2001), and, in the laboratory, they may be reluctant to descend to the height at which computer equipment is presented. Also, members of this species form strong social bonds with various group members (Fragaszy, Visalberghi, \& Fedigan, 2004), making it difficult to isolate individuals for testing, and such isolation may lead to agitation in these monkeys. Despite the strong bonds that capuchins form with other group members, their dominance hierarchy is relatively flexible in comparison with that of other primate species (Fragaszy et al., 2004). This may prevent investigators from separating capuchins for lengthy test sessions, since doing so may disrupt social harmony. Finally, capu- chins are very active, spending much time manipulating objects, foraging, interacting with others, and traveling (Fragaszy et al., 2004; Ross \& Giller, 1988). This activity budget can conflict with the level of focused attention required to engage in computerized test sessions of reasonable length.

Below we describe how we addressed and overcame these potential complications during the application of the Language Research Center's Computerized Test System (LRC-CTS; Richardson, Washburn, Hopkins, SavageRumbaugh, \& Rumbaugh, 1990) to a newly formed social colony of capuchins. The same collection of computers and other electronic equipment used with rhesus monkeys, great apes, and humans was used with these monkeys. However, several elements of apparatus and facility design and colony management were manipulated to produce an efficient testing environment.

\section{METHOD}

\section{Subjects}

The colony consisted of 9 tufted capuchin monkeys ranging in age from 2 years to 16 years. All 9 animals were originally housed together as one large social group. However, partway through training, the monkeys were divided into two smaller groups to prevent aggressive interactions between 2 adult males.

\footnotetext{
Materials

LRC-CTS. We used the LRC-CTS, a hardware and software package comprising a general-purpose computer, a color display monitor, a digital gamepad/joystick, external speakers, and a pellet dispenser linked to a digital I/O board within the computer through
} 
a solid-state relay board (see Table 1; Richardson et al., 1990; Washburn \& Rumbaugh, 1992). This system was originally devised to provide individually housed rhesus monkeys with 24-hour access to computerized tasks (the equipment was contained within clear Lexan enclosures). The test system has since been used to study many psychological processes, including attention, categorization, memory, numerical judgment, spatial cognition, self-control, and uncertainty monitoring (e.g., Rumbaugh \& Washburn, 2003; Washburn \& Rumbaugh, 1992).

Adaptation of materials. In order to adapt the LRC-CTS for use with the capuchins, a number of changes were made to the testing apparatus and procedures. These changes were necessary because of the differences both in housing conditions and in the behavioral tendencies of capuchins in comparison with other primate species exposed to this testing environment - primarily, chimpanzees and rhesus monkeys. The changes in testing equipment involved two major modifications. The first involved outfitting the capuchins' living quarters with banks of test enclosures, in which individuals could be temporarily isolated from the social group. The second major modification necessary to adapt the LRC-CTS for these monkeys was the assembly of mobile test systems that could be easily attached to and detached from test enclosures during test sessions, without risk of damage to the equipment by the animals. We describe each of these changes below.

We modified the existing colony rooms to create 150 - to $200-\mathrm{m}^{3}$ test areas, to which the monkeys did not have free access (Figure 1C). Each test area contained four $1-\mathrm{m}^{3}$ stainless steel mesh test enclosures that were suspended from a stainless steel mesh wall that was approximately $1 \mathrm{~m}$ above the floor, and were positioned approximately $0.5 \mathrm{~m}$ apart (see Figure 1C and Figure 2). Rectangular openings in the wall allowed monkeys to enter the test enclosures directly from their living quarters, and vertically sliding doors allowed experimenters to put monkeys in the enclosures for testing (see the Procedures section, below).

In addition to adequate testing areas, we needed an efficient method for providing these monkeys with access to computerized test systems. Because of this species' behavioral repertoire, great care had to be taken when designing apparatuses to hold and protect the test equipment. Capuchin monkeys are destructive foragers, a behavioral trait that lends to their success in the wild (Fragaszy et al., 2004); in captivity, this characteristic is manifested in capu-



Figure 1. The capuchin facility. In addition to (A) indoor and (B) outdoor animal living quarters and (C) animal testing enclosures, this building also contained (D) equipment storage and (E) husbandry areas.

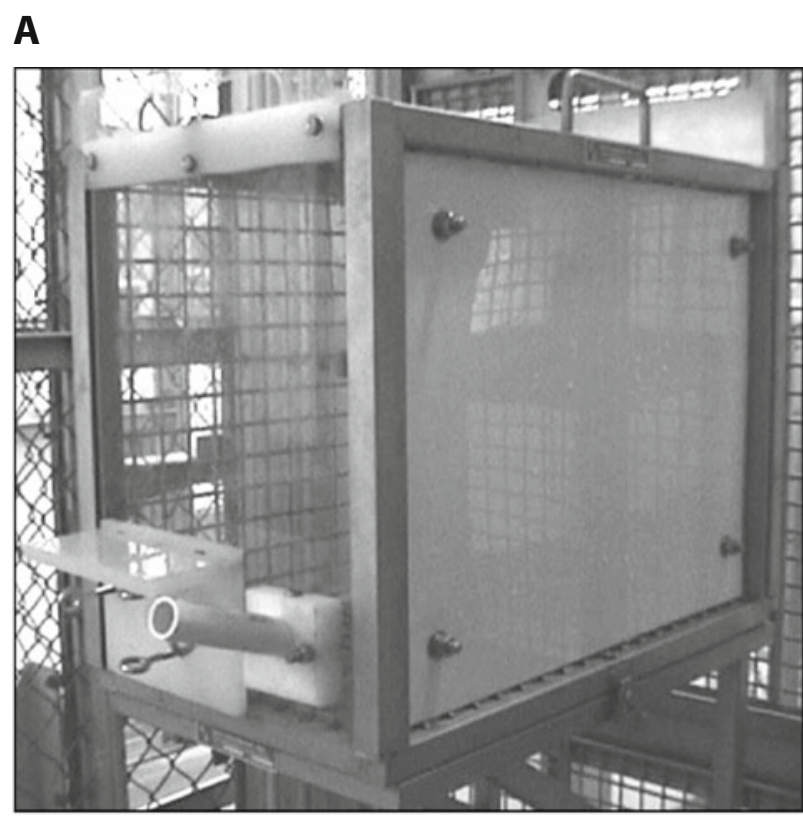

B

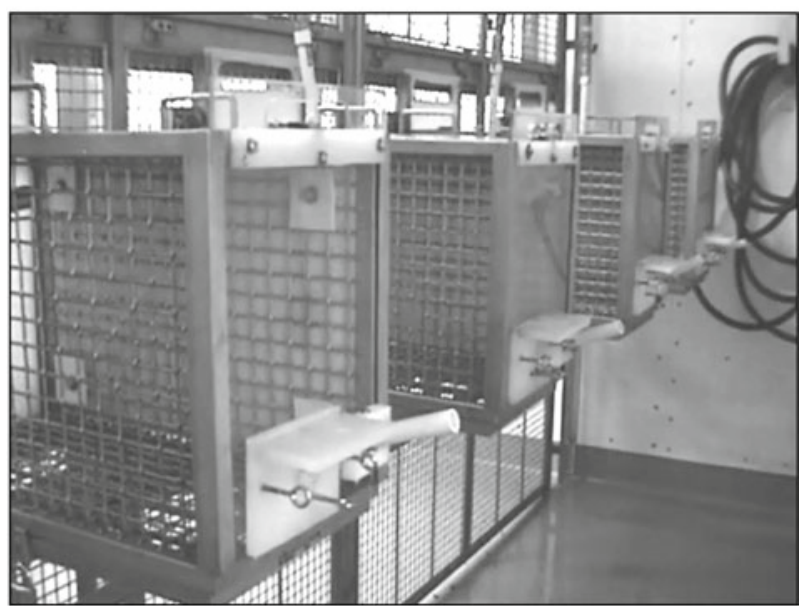

Figure 2. The capuchin test enclosures. (A) Each enclosure was outfitted with an opaque barrier on one side to prevent visual access between monkeys in adjacent enclosures. (B) Four side-byside enclosures were mounted perpendicular to a stainless steel mesh wall that separated the testing area from the capuchin living quarters.

chins' constant inspection and manipulation of the artificial environment, including testing facilities. Therefore, apparatuses were built to both protect the equipment and allow animals to interact with the test system.

The apparatuses and test system were mounted to utility carts so they could be removed from the colony room during cleaning; thus, we could use the test area for both computerized and noncomputerized research. A color monitor, a set of external speakers, and a pellet dispenser were located on the top shelf of the utility cart (Figure 3A). The pellet dispenser was fixed in place by a solid plastic mount, and a funnel with flexible tubing was mounted below the "head" of the dispenser in order to aid in pellet delivery (Figure 3B). A personal computer outfitted with a digital I/O card and relay board (to control the dispenser) and a digital gamepad was located on a lower shelf of the cart. 
Table 1

Components of the Capuchin Monkey LRC-CTS

\begin{tabular}{|c|c|c|}
\hline Component & Brand and Model & Details \\
\hline Test enclosure & $\begin{array}{l}\text { Britz-Heidbrink nesting box } \\
\text { (mounted to Flexagon panel) }\end{array}$ & $\begin{array}{l}\text { Stainless steel, welded wire, } \\
35 \mathrm{~cm}(\mathrm{w}) \times 60 \mathrm{~cm}(1) \times 46 \mathrm{~cm}(\mathrm{~h})\end{array}$ \\
\hline Utility cart & Bretford BP42-P4 & $\begin{array}{l}\text { Polyethylene } / \text { PVC, } \\
46 \mathrm{~cm}(\mathrm{w}) \times 61 \mathrm{~cm}(\mathrm{l}) \times 107 \mathrm{~cm}(\mathrm{~h})\end{array}$ \\
\hline Monitor & $\begin{array}{l}\text { Compaq V700 } \\
\text { (and other similar models) }\end{array}$ & $\begin{array}{l}43 \text {-cm color display, } \\
800 \times 600 \text { pixel resolution }\end{array}$ \\
\hline Computer & Compaq DeskPro & $\begin{array}{l}\text { Intel Pentium II processor, } \\
\text { Microsoft Windows } 98\end{array}$ \\
\hline Pellet dispenser & Life Science Associates RM45 & Built-to-suit 45-mg pellets \\
\hline Pellets & Bio-Serv F0159 & $\begin{array}{l}\text { 45-mg, grain-based, dustless } \\
\text { precision pellets, banana flavored }\end{array}$ \\
\hline Gamepad & Logitech Precision & $\begin{array}{l}\text { Digital/USB, 4-cm stainless steel rod } \\
\text { mounted on the directional button }\end{array}$ \\
\hline Speakers & $\begin{array}{l}\text { CyberAcoustics CA-2014 } \\
\text { (and other similar models) }\end{array}$ & $\begin{array}{l}\text { 2-piece amplified stereo desktop } \\
\text { system, } 3 \mathrm{~W}\end{array}$ \\
\hline Relay board & Keithley Instruments SRA-01 & 8-channel, solid-state module \\
\hline $\mathrm{I} / \mathrm{O}$ card & Keithley Instruments PIO-12 & 12-channel, digital \\
\hline
\end{tabular}

During testing, the cart was situated approximately 12 in. from the test enclosure, with the monitor centered directly in front of the monkey (Figure 3C). Custom-built apparatuses holding the gamepad and pellet tube were located on the surface of the test enclosure that faced the utility cart. These apparatuses allowed the monkeys to interact safely with the test system. Each apparatus consisted of a clear Lexan panel outfitted with opaque polyethylene and stainless steel components that were designed to hold the gamepad and pellet tube during testing (Figure $3 \mathrm{C}$ ). The components were designed so that the gamepad and pellet tube, which were both attached to the utility cart, could be quickly connected to and disconnected from the test enclosure (Figure 3D). The joystick portion of the gamepada stainless steel rod attached to the directional button-protruded through an opening in the Lexan panel, where it was accessible to the
A

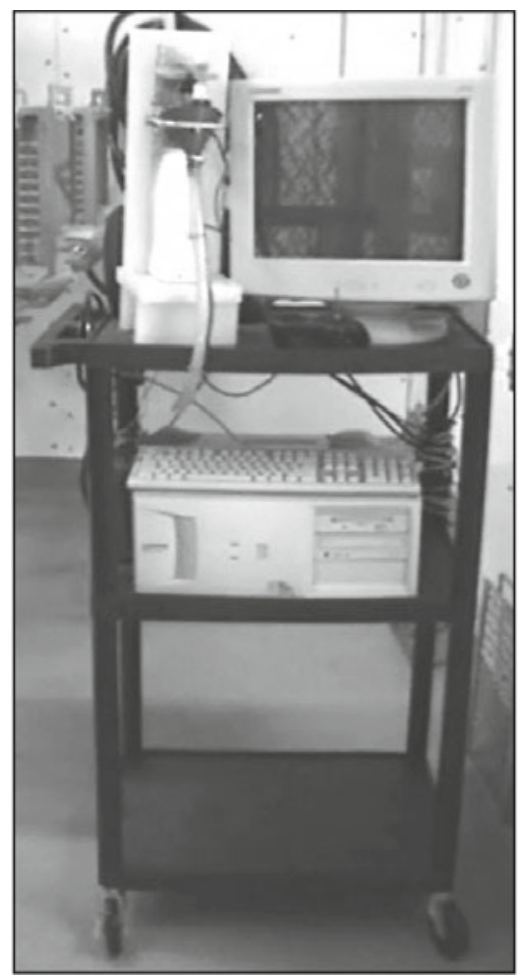

B

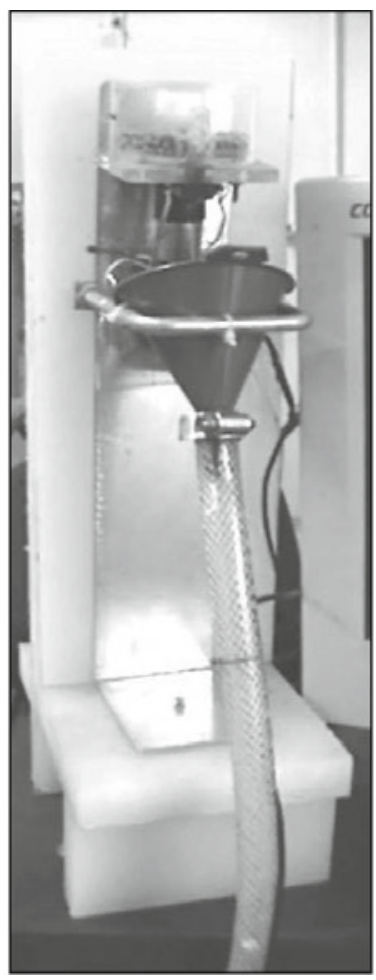

C



D

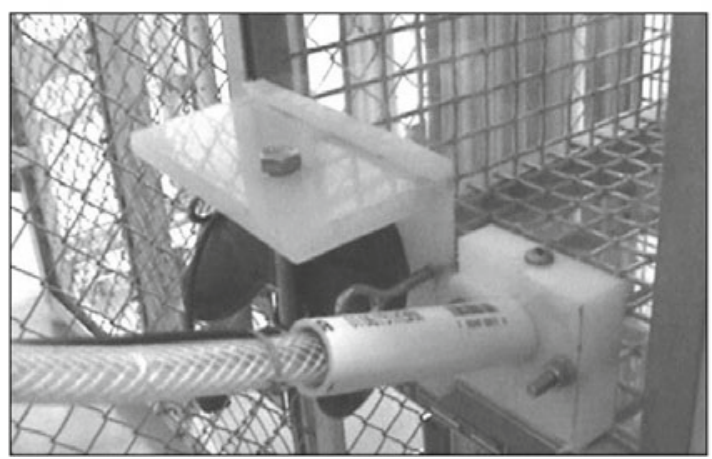

Figure 3. The capuchin LRC-CTS. (A) The computer and other electronic equipment was stored, transported, and employed on a utility cart. (B) The pellet dispenser was fixed to the top shelf of the cart by stainless steel and polyethylene components. (C) The dispenser fed into a plastic funnel and rubber tube that led to a small container on the Lexan door of the test enclosure. (D) The gamepad and pellet tube could be easily attached to and detached from stainless steel and polyethylene mounts on the test enclosure. 
monkey. The pellet tube was nested in a section of PVC tubing that protruded from the Lexan panel at an upward angle (Figure 3D). The PVC tubing led to an opening in the Lexan panel, where monkeys could access earned pellets. A lip in the opening prevented pellets from dropping into the test enclosure and being lost through the wire mesh at the bottom of the enclosure.

Since each test enclosure was close in proximity to other test enclosures and to the living quarters, visual barriers were needed in order to prevent potentially distracting social interaction between monkeys in these areas. For this reason, a side-mounted, opaque polyethylene panel was used in the final version of the test enclosure (Figure 1A). One panel was located on the same side of the enclosure as the pellet tube in order to provide additional protection from forceful manipulation of this piece of test equipment, in addition to preventing visual access to monkeys in adjacent test enclosures. The vertical sliding doors that separated the test enclosures from the rest of the living quarters also consisted of opaque polyethylene, and these panels prevented visual access to individuals that were not isolated for testing and remained in either the indoor or the outdoor living quarters.

\section{Software Applications}

All computerized tasks were written in Microsoft Visual Basic. These programs all involved a cursor (small solid red circle) that monkeys could control by manipulating a digital joystick with their hands. Monkeys were trained to use the cursor to make psychomotor and conceptual responses on the computer screen in a battery of four computerized tasks. The two psychomotor tasks were called side and chase. These tasks were used to ensure that monkeys knew how to control the cursor on the screen. In the side task, monkeys moved the cursor to illuminate portions of the computer screen; in the chase task, individuals moved the cursor into contact with moving targets. The two conceptual tasks in the training battery were matching to sample (MTS) and two-choice discrimination (discriminate) - tasks traditionally used in psychological research (see also Richardson et al., 1990). The tasks were presented seamlessly, meaning that completion of one task within a training session initiated the presentation of the next task.

For all tasks, the background color of the screen was white, and stimuli consisted of either solid-colored shapes or multicolored clip-art images. Task colors could be changed as needed to accommodate any specific visual-color capacities/deficits of the participants (capuchin monkeys exhibit a color vision polymorphism that results in a range of possible color-perception phenotypes across animals; Jacobs \& Deegan, 2003). However, we did not make use of this option, because most animals solved the tasks in the original form. All tasks also included a variety of settings that could be easily adjusted by the experimenter at program initiation in order to make the tasks either easier or more difficult, which aided in training the animals to criteria. For example, the experimenter could adjust the surface area of stationary targets, the speed of moving targets, or the number of accurate responses required for meeting success criteria. The experimenter could also choose to present only some of the tasks, rather than the full battery, so that monkeys did not have to repeat tasks that they had already mastered. We considered an animal to be computer trained when it could progress, within a single 120-min session, through the side, chase, MTS, and discriminate tasks, meeting the following success criteria for all tasks: side $=5$ consecutive trials at each target size, with a response time of less than $5 \mathrm{sec}$ each; chase $=20$ consecutive trials at each target size, with a response time of less than $10 \mathrm{sec}$ each; MTS = $80 \%$ correct matching performance over the most recent 50 trials; and discriminate $=80 \%$ correct (Trials 2-6) discrimination performance over 20 novel problems.

\section{Procedures}

Since capuchins were housed with multiple conspecifics, methods were devised to efficiently isolate individuals for computerized test- ing. Once separated from the group, monkeys also needed to willingly participate in testing. Thus, the colony's feeding and activity schedules were adjusted to maximize their motivation to participate.

Separation training. Across three training phases, we shaped the animals to enter test enclosures in the absence of an experimenter, then to enter test enclosures to receive food rewards held by an experimenter, and finally to be confined in the enclosures by an experimenter. In Phase 1, we habituated the monkeys to the test enclosures by allowing free access to the test enclosures, which were stocked with normal diet and treat foods throughout the day. This phase lasted for approximately 4 weeks, although this duration could have been much shorter. During Phase 2, monkeys were coaxed into the enclosures with food items held by the experimenter. Most of the monkeys would enter open test enclosures within a week. We then introduced the interior enclosure doors, gradually reducing the amount of open door space from $75 \%$ to $25 \%$ (just enough room for the monkey to fit under the door). The doors were designed with pin notches that allowed them to be locked in place at these varied heights. Finally, in Phase 3, we confined the monkeys in the test enclosures. At first, the monkeys were rewarded and immediately released, but later, we gradually increased the amount of time they were locked in the enclosure from 1 to $120 \mathrm{~min}$. During training sessions of greater length, monkeys received time-consuming enrichment items (stuffed tubes, Kong-brand toys, frozen treats, etc.) while in the enclosures. This final phase took somewhat more time to complete than did the previous steps, because monkeys were initially uncomfortable being isolated from their social group, as evidenced by increased vocalization and manipulation of the exit.

Provisioning. During the initial establishment of the colony, the capuchins had nearly constant access to a variety of foods. Feedings occurred three times a day and provided each animal with a total of approximately $500 \mathrm{Kcal}$. Therefore, the original feeding routine allowed much food to remain in the home enclosure between feedings. This feeding routine posed no problem during the initial phase of separation training, when the capuchins were required to go into wide-open test enclosures to accept a piece of highly preferred food without being locked in. However, as training progressed, this method became more problematic. During the phase in which the monkeys were required to enter and remain inside test enclosures with doors already in place, the value of the treats available inside the enclosures likely decreased in comparison with the ordinary food remaining in the home cage. To keep the capuchins motivated to enter the test enclosures, we adjusted the feeding schedule by limiting the monkeys' free access to food to more defined periods throughout the day. We did not, however, restrict their overall caloric intake.

We first altered the feeding routine by delaying the morning meal until after box training had taken place. Thus, during the training session, the monkeys could only receive food in the early morning by entering a test enclosure. However, the overall amount of calories that the monkeys were allowed to consume remained the same after this procedural change, because they were always fed after separation training. This feeding routine remained in place throughout the remainder of separation training and into the beginning of the computer training.

As training sessions reached 90 to $120 \mathrm{~min}$ in length, the morning and early afternoon meals were combined, due to time constraints. However, the overall amount of food given to the monkeys remained the same. The monkeys also continued to receive treats while confined inside the test enclosures, including nutritionally balanced food pellets that could be earned by working on computerized tasks. As the computer training phase progressed, the capuchins were reluctant to continue working for these pellets beyond a short period of time. Therefore, we altered the monkeys' feeding regimen once more. This change consisted of allowing the monkeys $2 \mathrm{~h}$ to feed on their evening meal, and then removing any remaining food items from the home enclosure at the end of the evening, when the caretakers left for the night (at approximately 6:00 p.m.). Thus, the animals could eat ad libitum prior to retiring, but food was not 
available several hours prior to the training session. However, it is important to note that none of these modifications were equivalent to reducing the animals in body weight, as is sometimes done in other situations or with other species. Body weight was maintained, as was the daily caloric intake. The changes pertained primarily to when calories were consumed. This feeding regimen remained relatively unchanged throughout the remainder of computer training. Thus, the final daily capuchin diet was as follows: at 9:00 a.m., small treat (e.g., a grape; $\leq 10 \mathrm{cal}$ ) for entering the test enclosure; from 9:00 to 11:00 a.m., 0.125 to 0.5 cup of food pellets $(45-150 \mathrm{cal})$; at 11:00 a.m., large treat to reward computer work (e.g., preferred fruit; 20-30 cal); from 11:00 a.m. to 12:00 p.m., small meal (e.g., miscellaneous vegetables; 50-100 cal); and from 4:00 to 5:00 p.m., large meal (i.e., fruit, vegetables, and monkey chow; 100-150 cal).

\section{RESULTS}

\section{Separation Training}

Eight of the 9 monkeys learned to enter the test enclosures and engage in a task for up to $120 \mathrm{~min}$. The 1 monkey that was not successful in this respect rarely allowed herself to be confined in an enclosure. For the successful monkeys, the training process required approximately 20 weeks. The majority of this time (approximately 10 weeks) was needed to put the monkeys at ease with being confined in the test enclosures. Thus, relatively little time was needed to train them to enter the enclosures (approximately 4 weeks), and little time was needed to increase the amount of time they would remain inside the enclosures (approximately 6 weeks).

Some procedural elements that were changed during separation training were more influential than others. Splitting the colony into two separate social groups had an immediate impact on the researchers' ability to separate individual monkeys into test boxes. This made it much easier to close the door behind an animal, because fewer group members were attempting to enter the enclosure at one time. Delaying the morning meal until after the training session was complete also expedited the separation training process. This presumably increased the monkeys' motivation to obtain food prior to the training session, making the treats offered during training more enticing.

\section{Training Tasks}

Seven of the 9 monkeys met the success criteria on all four computer tasks. The 2 monkeys that did not reach success criteria - Gambit and Lily (see Figure 4) — did not advance beyond the side task. Of the 7 successful monkeys, most were able to reach the final training criteria within 15 weeks. However, 2 of those monkeys-Drella and Logan - required substantially more time than did the others, because they sometimes ceased to work on computerized tasks for multiple consecutive weeks (Figure 4). By the time each of these 7 monkeys had reached the final success criteria, each was completing 500 to 1,000 training trials within a 2 -h session.

Several monkeys made major advancements in their computer training, immediately following certain methodological events (Figure 4). For example, several monkeys advanced through three or more task levels soon after a major change in the feeding procedure (Week 9). As mentioned above, this change consisted of removing all food from the monkeys' living quarters several hours prior to the morning computer training session. Changes

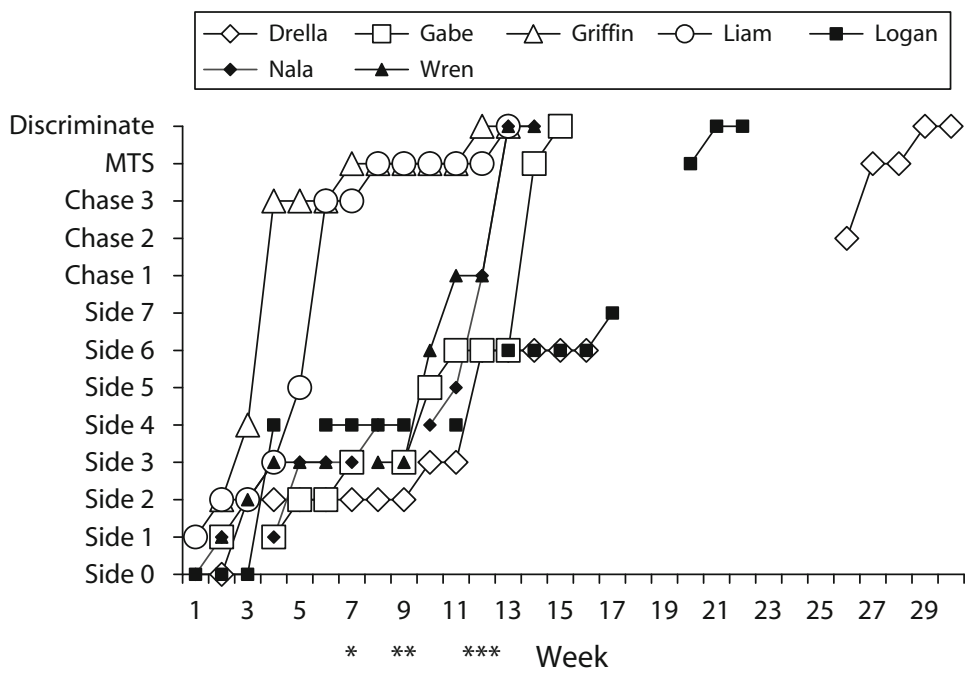

Figure 4. Cumulative advancements in training task performance. Only the 7 monkeys that fully completed computer training are represented here. Week numbers in which significant methodological changes occurred are denoted with asterisks: "The social group was divided into two smaller groups; ${ }^{* *}$ The feeding procedure was modified to prevent food consumption $15 \mathrm{~h}$ prior to test time; and ${ }^{* * *}$ The apparatus holding and protecting the gamepad and pellet delivery system was modified to prevent destructive manipulation by the monkeys, and opaque panels were installed on the sides of the test enclosures to prevent visual access to other monkeys. 
in work persistence were immediately noticeable following this procedural change, in that several capuchins seemed more eager to work on the computerized training programs.

A few monkeys made additional advancements after the installment of the final (more robust) joystick-andpellet-delivery apparatus and the addition of opaque side panels (Weeks 12 and 13). Earlier versions of the joystick-and-pellet-delivery apparatus comprised more flexible and accessible materials, which failed to prevent inappropriate contact with the equipment. This activity distracted the monkeys from their computerized tasks. Also, before the installment of the opaque side panels, little attention had been directed to the computer monitors because of distractions caused by nearby animals. Thus, controlling when the monkeys had free access to food and eliminating potential distractions from the computerized tasks were important early modifications to the testing procedure/system. These modifications resulted in faster, more reliable training.

\section{DISCUSSION}

Since the completion of the training battery, each capuchin has participated in several computerized experiments. These include studies of ordinal learning (Beran, Harris, et al., 2008), quantity conservation (Beran, 2008), reversal learning (Beran, Klein, et al., 2008), and perceived control over task order (Beran, Klein, Evans, Antworth, $\&$ Chan, 2007). The time required to complete each of these experiments ranged from 2 days to 4 weeks. In these experiments, the monkeys completed up to 1,500 trials in 120 -min (daily) test sessions. This level of productivity is comparable to that of the rhesus macaques at the Language Research Center, which is impressive, considering that the rhesus monkeys have 24-hour access to computerized tasks.

Additionally, we have continued to use the same basic method for separating individuals from their social group for other types of psychological testing, such as for manual tasks or tasks that present nondigital stimuli (Beran, Evans, Leighty, Harris, \& Rice, 2008). This procedure has also proven to be an asset for separating individuals for husbandry purposes. The repetitive use of the separation procedure has increased the monkeys' willingness to enter the test enclosures for all purposes and has reduced the overall amount of time required for separating the colony into individual enclosures to 5-10 min.

The outlined combination of manipulations to the capuchins' colony-room design, test hardware and software design, daily routine, and diet resulted in an excellent balance of labor input by the experimenters and data output by the monkeys. The elements that appeared to be most important for the monkeys' separation training success included limiting the number of animals that had access to the entrance of a test enclosure at one time and restricting access to nontest foods until after the completion of the training and testing sessions. This procedure as a whole was instrumental in allowing researchers to quickly recruit an animal for research and provide that animal with access to research materials.

Computerized training success was also influenced by major changes in the feeding schedule. In addition, the monkeys proceeded through training more quickly when they were prevented from manipulating test equipment in ways that were irrelevant to the tasks and when they could not interact with other monkeys in the vicinity of the test area. The computerized testing environment as a whole was successful in allowing researchers to obtain a large volume of data from motivated research subjects, and it allowed experiments to be conducted in a matter of days or weeks.

Despite these successes, this method may be improved further by some additional changes in the procedure or equipment. For example, some labor is required for transporting the mobile test apparatus in and out of the testing area. Leaving the apparatus in the testing area when not in use, without the potential for damage related to husbandry procedures, would further reduce the amount of effort required for setting an animal up on a computerized task. In our current laboratory space, this was not an option, due to the necessity of cleaning nearby stationary living areas, and because of our interest in using the test areas for both computerized and noncomputerized research. But in a different laboratory, perhaps some version of the method used by Richardson et al. (1990) to provide the rhesus monkeys with access to stationary, computerized test systems would prove more efficient.

Additionally, some small changes in procedure and equipment may allow us to use the current method to assess cooperative or competitive behavior in these monkeys, either in manual or computerized tasks. Finally, with some additional modifications and further training of the monkeys, we could use this variation of the LRCCTS to collect physiological and perceptual correlates of behavior, such as hormonal assays and eyetracking data. With the present method, we can efficiently assess a vast assortment of capuchin cognitive and behavioral capacities; but with these potential changes, we could expand the capabilities of the test system in order to address an even wider range of interesting and important empirical questions.

\section{AUTHOR NOTE}

The capuchin monkeys and this program of research are supported by National Institute of Child Health and Human Development Grant HD-38051 and the College of Arts and Sciences of Georgia State University. All applicable institutional rules and regulations regarding animal care and use have been followed in the care and testing of the monkeys. The experiments complied with all laws of the United States of America. The authors thank John Kelley for assistance with colony management, and Timothy Flemming, Emily Harris, and Sarah Hunsberger for assistance with training and computerized testing. Correspondence concerning this article should be addressed to T. A Evans, Language Research Center, Georgia State University, 3401 Panthersville Road, Decatur, GA 30034 (e-mail: theodore.evans@ gmail.com). 


\section{REFERENCES}

Beran, M. J. (2008). Capuchin monkeys (Cebus apella) succeed in a test of quantity conservation. Animal Cognition, 11, 109-116.

Beran, M. J., Evans, T. A., Leighty, K. A., Harris, E. H., \& Rice, D. (2008). Summation and quantity judgments of sequentially presented sets by capuchin monkeys (Cebus apella). American Journal of Primatology, 70, 191-194.

Beran, M. J., Harris, E. H., Evans, T. A., Klein, E. D., Chan, B., Flemming, T. M., ET AL. (2008). Ordinal judgments of symbolic stimuli by capuchin monkeys (Cebus apella) and rhesus monkeys (Macaca mulatta): The effects of differential and nondifferential reward. Journal of Comparative Psychology, 122, 52-61.

Beran, M. J., Klein, E. D., Evans, T. A., Antworth, R., \& Chan, B. (2007). Perceived control, motivation, and task performance in capuchin monkeys. In P. R. Zelick (Ed.), Issues in the psychology of motivation (pp. 171-185). Hauppauge, NY: Nova Science.

Beran, M. J., Klein, E. D., Evans, T. A., Chan, B., Flemming, T. M., HARris, E. H., ET AL. (2008). Discrimination reversal learning in capuchin monkeys (Cebus apella). Psychological Record, 58, 3-14.

Fortman, J. D., Hewett, T. A., \& Bennett, B. T. (2001). The laboratory nonhuman primate. Boca Raton, FL: CRC Press.
Fragaszy, D. M., Visalberghi, E., \& Fedigan, L. M. (2004). The complete capuchin: The biology of the genus cebus. Cambridge, UK: Cambridge University Press.

JaCOBS, G. H., \& DeEgan, J. F. (2003). Cone pigment variations in four genera of new world monkeys. Vision Research, 43, 227-236.

Richardson, W. K., Washburn, D. A., Hopkins, W. D., SavageRumbaugh, E. S., \& Rumbaugh, D. M. (1990). The NASA/LRC Computerized Test System. Behavior Research Methods, Instruments, \& Computers, 22, 127-131.

Ross, R. A., \& GILleR, P. S. (1988). Observations on the activity patterns and social interactions of a captive group of black-capped or brown capuchin monkeys (Cebus apella). Primates, 29, 307-317.

RumbaUgh, D. M., \& WASHBURN, D. A. (2003). Intelligence of apes and other rational beings. New Haven, CT: Yale University Press.

Washburn, D. A., \& Rumbaugh, D. M. (1992). Testing primates with joystick-based automated apparatus: Lessons from the Language Research Center's Computerized Test System. Behavior Research Methods, Instruments, \& Computers, 24, 157-164.

(Manuscript received June 13, 2007;

revision accepted for publication November 23, 2007.) 